\title{
Linx
}

Revue des linguistes de l'université Paris X Nanterre

11 | 1999

Typologie des langues, universaux linguistiques

\section{Typologie syntaxique et grammaire de texte}

\section{Bernard Combettes}

\section{OpenEdition}

\section{Journals}

Édition électronique

URL : http://journals.openedition.org/linx/893

DOI : $10.4000 /$ linx.893

ISSN : 2118-9692

\section{Éditeur}

Presses universitaires de Paris Nanterre

\section{Édition imprimée}

Date de publication : 1 décembre 1999

Pagination : 153-165

ISSN : 0246-8743

\section{Référence électronique}

Bernard Combettes, «Typologie syntaxique et grammaire de texte », Linx [En ligne], 11 | 1999, mis en ligne le 02 juillet 2012, consulté le 19 avril 2019. URL : http://journals.openedition.org/linx/893 ; DOI 10.4000/linx.893

Ce document a été généré automatiquement le 19 avril 2019.

Département de Sciences du langage, Université Paris Ouest 


\title{
Typologie syntaxique et grammaire de texte
}

\author{
Bernard Combettes
}

1 Le développement de la grammaire de texte conduit presque obligatoirement à poser la question des rapports qu'il convient d'établir entre les catégories pertinentes pour l'analyse discursive et le système linguistique propre à chaque langue. Indépendamment des problèmes spécifiques au champ particulier de l'étude de la textualité (délimitation des unités, fonctionnement des diverses marques, rôle du contexte, etc.), se présente de façon incontournable le problème de ce que l'on appelle ordinairement le «codage » des structures textuelles. La linguistique du texte -il en irait de même pour l'analyse du discours - rencontre obligatoirement la typologie, dans la mesure où il s'agit pour elle, au bout du compte, d'observer la mise en œuvre dans un cadre textuel des divers soussystèmes offerts par les langues. Cette question se pose avec d'autant plus de force que les objectifs de la linguistique du texte se sont de plus en plus éloignés de l'approche globale, qui s'intéressait aux relations des éléments à l'intérieur du texte, tentant de rendre compte de la cohérence générale, mais qui faisait trop souvent négliger le poids de la spécificité des systèmes linguistiques concernés. On peut constater, depuis un certain nombre d'années, le net développement d'une autre tendance, qui consiste à mettre l'accent sur des domaines particuliers, à limiter l'analyse à certaines familles et sousfamilles de marques ou de "notions" (anaphores, connecteurs, opposition des plans, modalisation, etc.) ; c'est en grande partie cette restriction de l'observation qui a tout naturellement conduit à s'interroger sur la nature, la place, l'importance, des « outils » fournis par les structures des langues, qu'il s'agisse de syntaxe ou de sémantique. Il convient aussi de remarquer que la linguistique textuelle a rapidement vu ses concepts et sa méthodologie se trouver appliqués à des langues de familles fort diverses, ce qui a permis d'éviter une vision trop limitée, et par là même biaisée, du problème qui nous intéresse ici. Avant d'illustrer par quelques exemples précis certaines questions soulevées par l'opération de codage, nous voudrions rappeler les principales prises de position, les principales réponses, que ce type de problématique a entraînées. 
2 Une première possibilité consiste à partir, si l'on peut dire, du «point de vue » de la typologie; dans ce cas, la liaison avec le domaine textuel et discursif peut être sentie comme une exigence, une nécessité, dès qu'il s'agit de passer à une étape d'explication, de "justification », des structures linguistiques observées et de leur répartition typologique : question souvent soulevée de la place et de l'importance que l'on accordera au fonctionnalisme dans une étude linguistique. Il peut s'agir par exemple d'apporter des arguments dans la définition et l'identification d'une " phrase de base » : c'est l'insertion dans tel ou tel type de contexte qui va permettre de considérer un schéma syntaxique comme marqué ou non marqué. Il peut s'agir aussi de rendre compte d'une évolution, du passage, pour une langue donnée, d'un type à un autre ; cette démarche est fréquemment utilisée, et on peut constater que la plupart des approches diachroniques développent l'idée qu'il est nécessaire de faire appel à des facteurs "externes", la prise en considération des seuls facteurs « internes ", des points d'équilibre et de déséquilibre du système ne suffisant pas à expliquer le changement. Parmi ces facteurs externes, d'ordre fort divers, le niveau textuel joue un rôle important, dans la mesure où il permet de mettre en relation faits de langue et aspects cognitifs. Dans une telle perspective, la dimension textuelle est plutôt considérée comme une sorte d' "aide », de complément, à la typologie : le texte exploite, en quelque sorte, un matériau qui a sa structuration et ses règles de fonctionnement propres. On retrouve ainsi une démarche somme toute classique, qui consiste à reconnaître, à dégager, des valeurs en langue, qui se spécialisent dans divers emplois contextuels, dans des « effets » de discours. Le niveau syntaxique se prête particulièrement bien à cette approche : le système linguistique offre un ensemble de constructions différentes de même contenu propositionnel, et les différences dans l'ordre des constituants peuvent, dans de nombreuses langues, être exploitées par exemple pour traduire, avec plus ou moins de précision et de clarté, les divers aspects de la présentation des référents (introduction d'un référent nouveau, réactivation d'un référent déjà évoqué, marquage d'un référent saillant, etc.). Dans une telle perspective, les «nécessités» discursives n'ont pas d'influence, d'impact, sur les structures syntaxiques ; comme nous l'avons relevé plus haut, cela n'empêche pas de s'appuyer sur cette relation langue / texte pour parler de tour marqué ou de tour non marqué ; cela n'interdit pas non plus de parler d'iconicité et d'interpréter cette relation en termes d'homologie, mais il est évidemment tout à fait possible de privilégier l'arbitraire du signe et de ne présupposer aucune ressemblance systématique entre le plan de la langue et celui du discours.

3 Un autre point de vue consiste à se placer délibérément du côté du texte ; il ne s'agit plus de « justifier » l'existence ou le statut de tel ou tel fait de langue, mais de rendre compte des règles de fonctionnement des marques linguistiques de la cohérence. Ce qui est alors tenu pour primordial, ce sont, disposés dans une sorte d'emboîtement, le type d'action langagière, le type de discours, la mise en texte, l'organisation interne ; faire l'hypothèse d'une valeur en langue préalable à l'actualisation dans le discours n'a alors guère d'intérêt, dans la mesure où l'on estime que les seuls facteurs pertinents relèvent du texte et qu'ils conditionnent, en quelque sorte, le système linguistique. L'analyse bien connue que proposent Hopper et Thompson (1980) pour rendre compte de la transitivité, peut être considérée, dans une large mesure, comme une illustration de cette tendance; en effet, c'est bien la nécessité de coder l'opposition des plans (premier plan / second plan), présentée comme universelle, qui va déterminer l'ensemble des propriétés de la «transitivité » forte, réunion de caractéristiques conçue comme une sorte de 
conséquence du marquage du premier plan. Le but de ce type d'étude n'étant pas de rendre compte du système de la langue, mais d'analyser des régularités d'ordre textuel, on comprend que la liaison avec la typologie ne soit ni directe, ni étroite. Ceci est aggravé, pourrait-on dire, par le fait que les notions textuelles sont souvent présentées comme des universaux, qu'il suffirait de mettre en relation avec les "outils", syntaxiques, sémantiques, offerts par les diverses langues. Certes, comme le suggère Myhill (1992), cette approche peut aider à une analyse typologique plus fine : telle structure reconnue comme "passif » dans une langue est-elle exactement identique à telle autre que l'on interprète comme passif dans une autre langue ? Des différences dans le fonctionnement textuel peuvent, indirectement, faire réviser un classement, une caractérisation typologique, mais ceci n'est qu'une conséquence quelque peu marginale de la prise en compte des faits de langue. Force est de constater que font encore défaut les études comparatives qui permettraient d'établir ce que Myhill nomme la «typologie discursive » : il conviendrait en effet de pouvoir opposer les systèmes et les sous-systèmes linguistiques en prenant comme critère déterminant la façon dont ils assurent le codage des catégories textuelles.

Quelle que soit la position adoptée, il nous semble toutefois que la prise en compte du texte ou du discours s'accompagne presque toujours de ce que l'on pourrait caractériser comme un "glissement", qui fait que le texte n'est guère considéré dans sa spécificité, mais devient une unité comme les autres, le lieu où se contextualisent les structures linguistiques; c'est sur la nécessité de mieux prendre en compte cette spécificité que nous voudrions insister à présent : quel que soit l'objectif visé, la mise en relation des deux domaines (typologie linguistique, catégories discursives) ne peut s'opérer de façon satisfaisante que si les notions textuelles sont elles-mêmes considérées comme structurées et formant système, avec toutes les conséquences que cela comporte. Cette occultation de la spécificité du texte est sans doute due, en grande partie, au fait que ce que nous avons présenté jusqu'ici comme la mise en rapport de deux domaines met en réalité en jeu trois dimensions : ce sont en fait des catégories et des opérations cognitives qui se trouvent concernées par le codage, le texte ne constituant plus que le champ d'application de cette activité. Givon (1992) souligne explicitement la place de chacune des instances: "In actual communicative behavior, the grammar does not directly interact with the text. Rather, the grammar interacts with the mind that produces or interprets the text.» (p. 5), ce qui, d'un point de vue méthodologique, pourrait se traduire, assez schématiquement, par la position suivante : les catégories cognitives qui sont en relation avec le système linguistique ne peuvent être atteintes qu'à travers leurs réalisations concrètes dans les textes; la dimension textuelle devient indispensable dans la mesure où elle permet l'observation du fonctionnement de ces catégories, où elle donne le moyen de les définir avec précision, par la prise en compte du contexte, en particulier. Il n'est alors pas étonnant de constater une grande hétérogénéité dans les caractéristiques, les «notions» textuelles qu'il conviendrait de mettre en relation avec des faits de langue. Nous pouvons ainsi considérer les paramètres énumérés par Myhill, paramètres qui permettraient de rendre compte de telle ou telle construction en contexte (la séquence SVX opposée à XVS, par exemple) : ils concernent des caractéristiques aussi diverses que la récence de la mention du référent, le fait qu'il y ait ou non séquence temporelle dans le texte, critères que l'on peut renvoyer nettement au domaine du texte, mais ils englobent aussi le caractère "humain" d'un syntagme nominal, le caractère " affecté » du patient, autant de traits qui relèvent davantage du domaine sémantique que de la dimension strictement discursive. C'est bien en fait la relation langue / cognition 
qu'il s'agit de mettre en lumière; le texte est certes l'unité qui conduit aux phénomènes de succession, de linéarisation, qui met obligatoirement en jeu les activités de mémorisation, et qui, par là même, exige du locuteur une maîtrise de la gestion de la « connaissance partagée », du niveau informatif, mais les catégories qui pourraient être propres à la dimension textuelle sont souvent perçues et présentées comme le simple regroupement de plusieurs indices, différents selon les types de langue, qui serviraient ainsi à coder des « notions » universelles. Dans leur approche de la transitivité, à laquelle nous avons fait allusion plus haut, Hopper et Thompson vont dans ce sens, lorsqu'ils définissent le prototype de l'énoncé transitif comme celui qui combine des caractéristiques relevant de la valence (présence du couple agent / objet), de l'aspect verbal (aspect télique; vision sécante / vision globale), de la sémantique des relations syntaxiques (objet affecté), de la sémantique du procès (action ou événement), etc. La typologie est concernée dans la mesure où les marques linguistiques qui renvoient à ces divers types d'information ne sont évidemment pas identiques dans toutes les langues; ce qui mériterait discussion et qui est loin d'aller de soi, c'est la liaison qui est établie entre ce concept de transitivité et celui de l'opposition des plans, liaison qui aboutit à une quasi assimilation de l'un à l'autre: les énoncés à fort degrés de transitivité permettent le codage du premier plan, ceux qui présentent un faible degré de transitivité sont réservés au second plan. Même si une telle conclusion correspond sans doute dans les faits à une constatation correcte, il nous semble difficile de considérer les divers aspects de la cohérence textuelle comme un donné évident, inhérent à tout discours, dont la définition resterait aussi constante et aussi universelle que les facteurs sémantiques (action vs événement; télicité du procès, etc.) qui sont pris comme base de la transitivité. De ces paramètres aux oppositions textuelles, le pas est vite franchi : «Whitout the connection to a communicative function, the separate components of the transitivity relationship have only an arbitrary relationship to each other ; we lack a reason why these semanticgrammatical components, rather than others, should be selected» (p. 280). Cette nécessité de "justifier" les divers composants de la transitivité conduit à rechercher dans l'opposition premier plan / second plan le «point fixe » pour lequel toute langue doit fournir un codage. Les variations que l'on accepte de reconnaître dans ce domaine textuel, même si elles apparaissent comme dépendant de certaines règles, sont renvoyées aux contraintes de la communication, de la situation, des choix du locuteur: "While we claim that the discourse distinction between foregrounding and backgrounding provides the key to understanding the grammatical and semantic facts we have been discussing, we also explicitly recognize that grounding itself reflects a deeper set of principles relating to decisions which speakers make, on the basis of their assessment of their hearers' situation, about how to prevent what they have to say » (p. 295).

5 Cette démarche, qui aboutit finalement, dans le souci de rassembler divers faits de langue sous une même fonction discursive, à dénier implicitement aux notions textuelles une structuration qui leur serait propre, ne diffère guère, dans son résultat, de l'autre point de vue qui consiste à partir du texte pour procéder à une analyse du codage. On pourrait s'attendre, dans ce cas, à ce qu'une attention particulière soit accordée aux différentes composantes de la cohérence, qui sont finalement l'objet d'étude ; en réalité, ici encore, la prise en compte de la dimension cognitive, considérée d'une façon trop indépendante, fait souvent négliger la spécificité du texte. Pour reprendre l'exemple de l'opposition des plans, on pourra constater qu'une analyse comme celle que propose Reinhart (1984), dans le prolongement des définitions de Labov, présente la dichotomie premier plan / second plan comme une donnée universelle qu'illustreraient tous les récits, tous les textes 
narratifs ; cette universalité est justifiée par le caractère apparemment général, dans le domaine de la perception, de la distinction forme / fond, avec laquelle, effectivement, l'opposition des plans peut être mise en rapport. Comme dans l'approche évoquée plus haut, le statut que l'on accorde aux catégories cognitives semble s'étendre au texte luimême, et la seule variation reconnue sera celle des catégories linguistiques propres aux divers types de langues. Que l'on interprète ensuite le codage comme une illustration des principes de l'iconicité, en essayant de trouver jusqu'aux niveaux «inférieurs" de l'analyse linguistique l'influence de la composante cognitive (Dressler, 1996), que l'on défende au contraire l'arbitraire du signe (Prince, 1988), que l'on adopte des positions intermédiaires, qui reconnaissent une certaine autonomie au niveau linguistique (Dubois, 1987 ; Hopper, P., 1987 ; O'Dowd, 1990 ; Payne, 1987, 1994 ; Durie, 1994 ; Hopper, R., 1998), le résultat final nous semble identique : les relations, les déterminations que l'on tente de mettre en lumière se situent en réalité entre la langue et le domaine cognitif, alors que la spécificité des structures textuelles n'est guère prise en compte.

6 C'est sur la nécessité de donner sa juste place au niveau textuel que nous voudrions insister à présent, en prenant comme exemple le cas de l'opposition des "plans » en français, et en essayant de montrer, dans une perspective historique, que ce ne sont pas seulement les marques linguistiques (en l'occurrence, ici, l'ordre des mots) qui peuvent varier, mais que la notion même de plan doit être "relativisée " et relève d'une structuration qui lui est propre. On peut en effet constater que, dans la plupart des textes narratifs de l'ancien et du moyen français, le second plan n'apparaît pas comme une entité autonome, qui aurait ses propres règles de fonctionnement, mais comme un ensemble de propositions placées sous la dépendance du premier plan, subordonnées, en quelque sorte, à la séquence narrative, au déroulement chronologique. Une progression thématique assez simple, prenant comme thème constant un personnage, va permettre d'introduire les divers éléments de la description; ce sont les verbes de perception, les verbes d'action, qui évitent de placer le référent nouveau, d'ordinaire indéfini, en position initiale. Ainsi, dans les deux passages suivants, les propositions de second plan sont-elles constituées de subordonnées relatives dépendant elles-mêmes de syntagmes nominaux « nouveaux », introduits par des verbes de premier plan qui ont pour sujet le personnage principal, en l'occurrence Lancelot:

Et tant a alé que il vint a une croiz de pierre qui ert au departement de deux voies en une gaste lande. Et il regarde la croiz quant il fu pres et voit par dejoste un perron de marbre ou il avoit lettres escrites. Et il resgarde vers la croiz et voit une chapelle moult ancienne, et troeve a l'entree unes prones de fer, et voit la dedenz un autel qui (...) (La Queste del Saint Graal, XIII ${ }^{\text {ème }}$ s.)

'et il a tant marché qu'il arrive à une croix de pierre qui était au carrefour de deux chemins dans une lande déserte. Et il regarde la croix quand il est tout près et voit à côté un bloc de marbre où il y avait des lettres écrites. Et il regarde vers la croix et voit une chapelle très ancienne, et il trouve à l'entrée des barreaux de fer, et il voit à l'intérieur un autel qui ..'

Si li avint une foiz tout par nuit qu'il ariva de lez une forest a l'oriere dou bois. Lors escouta et oi un chevalier venir a cheval (id.)

'Il lui arriva une fois pendant la nuit qu'il arriva près d'une forêt, en lisière du bois. Alors il écouta et entendit venir un chevalier à cheval.'

7 La dimension chronologique est ici importante; dans ce type de progression, de présentation de l'information, l'emploi de particules telles que et, lors, si, souligne le déroulement temporel des événements rapportés. L'introduction des référents nouveaux et, plus généralement, les unités de second plan qui vont leur être attachées, se trouvent ainsi sous la dépendance de la composante narrative du texte. Deux tendances se 
rencontrent alors : celle qui consiste, dans le domaine sémantico-référentiel, à ne pas commencer la proposition par un référent "nouveau», celle qui privilégie, dans le domaine syntaxique, l'ordre à verbe second. Dans le deuxième des exemples cités, il aurait été possible de construire l'énoncé de la façon suivante : Un chevalier vint à cheval ..., mais des progressions de ce type ne surviennent que rarement; ce que l'on pourrait analyser comme une rupture thématique est systématiquement évité par l'emploi des verbes de perception ou de mouvement, qui renvoient au premier plan du texte, et permettent de maintenir le schéma syntaxique habituel. Il faut remarquer que le type de texte, en l'occurrence ici la fiction narrative, remplit une fonction importante dans cette problématique ; ce que l'on pourrait appeler la primauté du narratif sur le descriptif est à rattacher au statut du personnage qui se trouve maintenu de façon continue dans tous les épisodes du récit et sert en quelque sorte de point fixe autour duquel s'organise la présentation des divers événements, en particulier la structuration, la hiérarchisation, de l'opposition des plans. Système de la langue et marques de la cohérence textuelle peuvent être ainsi mis en relation, le second plan correspondant effectivement à des phénomènes de "subordination" syntaxique, qu'il s'agisse de propositions subordonnées ou de constructions adjectivales; l'autonomisation progressive du second plan devra être mise en rapport avec une évolution dans la conception du personnage, qui perd certaines de ses caractéristiques d'organisateur textuel. Les oeuvres de moyen français font apparaître des alternances qui opposent au système que nous venons rapidement d'évoquer la possibilité d'introduire le second plan dans une proposition non dépendante ; ainsi, par exemple, dans le Roman de Jehan de Paris ( $\mathrm{XV}^{\text {ème }} \mathrm{s}$.), à côté de l'enchaînement :

Quant tout fut appresté, on alla a l'esglise, qui fu moult richement tendue de fleurs de lis, trouve-t-on :

Et quant il entra en la salle ... il se gecta a genoulz... La salle estoit toute tendue de moult riche tapisserie, et le ciel et le pavement aussi tout tendu, passage dans lequel le référent la salle (il est vrai déjà cité dans le contexte) apparaît comme sujet d'une proposition renvoyant au second plan.

La constitution de l'arrière-plan comme portion de texte «indépendante », possédant sa propre organisation, a sans doute été favorisée par un facteur relevant lui aussi de la structuration textuelle, qui réside dans le développement de la progression thématique « linéaire »; dans ce type de progression, le rhème, ou une partie du rhème, d'un énoncé est repris comme thème de l'énoncé qui suit dans le contexte immédiat. Cet enchaînement, fondamentalement différent de celui dont nous venons de citer quelques exemples, autorise l'introduction dans le texte de référents nouveaux après l'élément verbal, sans qu'il y ait nécessité de mettre en jeu un syntagme représentant le personnage principal. C'est un élément déjà évoqué et déjà décrit qui est utilisé comme point de départ de la proposition de second plan qui contient à son tour une nouvelle unité, etc. Ces passages, essentiellement descriptifs, sont donc bâtis sur une juxtaposition des diverses composantes de la réalité décrite, avec passage de l'une à l'autre; la dimension chronologique est alors abandonnée pour laisser la place à ce qui correspondrait davantage à un effet de liste, d'énumération. Le passage suivant, tiré d'une œuvre historique du XIII ${ }^{\text {ème }}$ siècle, la Conqueste de Constantinople, de Robert de Clari, montre bien comment s'opère la progression de l'information dans ce type d'organisation textuelle :

Aprés devant chu moustier de Sainte Sophie, avoit une grosse colombe qui bien avoit trois brachies à un homme de grosseur, et si avoit bien cinquante toises de haut; si estoit faite de marbre et puis de coivre par dessus le marbre, et estoit molt 
bien liée de bonnes bandes de fer. Lassus, sur le bout de chelle colombe, si avoit une pierre qui bien avoit quinze piés de lonc et autant de lé. Sur chelle pierre si avoit un empereur jeté de coivre, qui tendoit sa main vers paienisme.

'Après, devant cette église de Sainte Sophie, il y avait une grosse colonne qui avait bien trois brassées d'homme de grosseur, et elle avait bien cinquante toises de haut; elle était faite de marbre et puis de cuivre par dessus le marbre, et elle était bien entourée de bonnes bandes de fer. En haut, au sommet de cette colonne, il y avait un empereur fait de cuivre, qui tendait sa main vers les païens.' linéarisation : circonstant + verbe $+\mathrm{X}$, avec l'emploi de l'impersonnel (avoit: 'il y avait'), très fréquent dans ce type de progression. Le référent nouveau a la fonction syntaxique de séquence de l'impersonnel et se trouve «décrit » par l'adjonction de subordonnées relatives, de syntagmes adjectivaux ou participiaux, ce qui semble aboutir au même résultat que lorsqu'il s'agit d'une progression à thème constant; il faut cependant souligner que la disposition linéaire autorise beaucoup plus facilement le développement $\mathrm{du}$ second plan sous la forme de propositions indépendantes, comme c'est le cas dans l'exemple cité (si estoit faite de marbre .. et estoit molt bien liée...) : l'élément nouveau introduit par le verbe impersonnel est utilisé en fonction de thème constant d'un passage de second plan. L'absence de personnage principal dont la présence serait maintenue tout au long du texte favorise évidemment une telle construction des parties descriptives; on peut toutefois relever, dans les textes de fiction, différentes combinaisons qui font alterner les deux types de progressions thématiques, avec des degrés divers de dépendance de l'une à l'autre, comme dans :

Quant ilz eurent passé la première porte et furent sous le portail, le Cueur apperceut en la voulte du portail pendue une corbeille faite d'osier, ancienne et de vieille façon, laquelle pendoit à une chaigne d'or grosse comme le bras. Et en regardant qu'il faisoit ladicte corbeille, s'arresta et vit unes forces de fer enroullées qui estoit de la grandeur d'un pié et demi. Entre le taillant desquelles forces avoit une grosse poignée de cheveulx noirs ...

(René d'Anjou, Le Livre du Cuer d'Amours Espris, XV ème $\mathrm{s}$.)

'quand ils eurent passé la première porte et qu'ils furent sous le portail, le Coeur aperçut sous la voûte du portail une vieille corbeille pendue, faite d'osier, qui pendait à une chaîne d'or grosse comme le bras. Et comme il regardait cette corbeille, il s'arrêta et vit des ciseaux de fer rouillés qui étaient de la grandeur d'un pied et demi. Entre les lames de ces ciseaux, il $y$ avait une grosse poignée de cheveux noirs ...'

Les verbes de perception (aperçut, vit) correspondent à la progression à thème constant, alors que l'impersonnel permet l'insertion d'un thème linéaire, qui fait «oublier », pourrait-on dire, le thème initial (le Coeur), pour concentrer l'attention sur le second plan, sur la description de l'objet.

11 Même si l'on peut considérer que la progression à thème linéaire va dans le sens d'une certaine conception du second plan comme unité textuelle large, regroupant plusieurs propositions indépendantes du point de vue syntaxique, alors que la progression à thème constant ne peut guère déboucher sur une telle réalisation, il faut bien constater que ces deux types de disposition de l'information ont au moins une caractéristique commune, qui est d'assurer une cohérence textuelle " étroite ", qui voit son domaine restreint à un contexte de deux ou trois phrases. Chaque énoncé, dans un cas comme dans l'autre, est rattaché très explicitement à celui qui le précède, les «reprises » s'opérant par des répétitions d'expressions ou par des anaphores, pronominales ou démonstratives, qui ne soulèvent pas de problèmes d'identification. Il est donc sans doute nécessaire, pour expliquer l'émergence d'un second plan autonome, de se tourner vers un troisième type 
de progression, qui existe dès les plus anciens textes, mais qui se développe pleinement avec la production en français de discours informatifs et argumentatifs; il s'agit de la progression ordinairement dénommée "à thèmes dérivés ", ou à "hyperthème ", qui assure une cohérence beaucoup plus élargie, dans la mesure où elle peut structurer des portions de textes de plus en plus longues et, par là-même, isoler davantage le second plan du premier plan. Ce qu'il est intéressant d'observer, ce ne sont pas tant les occurrences d'un hyperthème "collectif", divisé en ses divers constituants, dans une sorte de « reprise partitive » (Les deux chevaliers... L'un... L'autre..., etc.), disposition qui ne fait guère sortir du cadre de la cohérence étroite, que les extraits dans lesquels l'enchaînement est assuré par des phénomènes sémantiques qu'il convient de rapprocher des inférences: les thèmes dérivés qui se suivent dans le passage de second plan correspondent à des référents dont l'existence est présupposée, de façon plus ou moins directe, par celle du référent auquel renvoie l'hyperthème. Dans le premier cas, la liaison thématique ne s'exerce que sur les groupes nominaux (hyperthème et thèmes dérivés); en revanche, lorsqu'il s'agit d'un fonctionnement inférentiel de la division en sousthèmes, on constate que c'est souvent l'événement rapporté par la totalité du premier énoncé qui va être la source des inférences, et qu'il est difficile de diviser nettement les énoncés qui constituent le développement du second plan en constituants thématiques et en constituants rhématiques, dans la mesure justement où c'est l'ensemble de chacune des phrases qui va se rattacher à l'énoncé hyperthème initial. Dans le passage descriptif suivant, qui relate différents aspects du lever du jour, le changement de plan est marqué, au début du texte, par l'utilisation de la progression à thème constant (alors ils virent l'air... ), qui rattache la description au contexte de gauche, de type narratif ; mais le second plan évolue ensuite sans qu'il y ait retour aux personnages, sans qu'il y ait non plus reprise des référents d'une phrase à l'autre. Quelques marques traduisent bien les enchaînements ( d'autre part, aussi, si), mais rien ne laisse attendre l'introduction de tel ou tel référent dans le texte :

Si virent lors l'air net et pur sans vent et sans nuée, et le jour gagnoit la nuit forment, en soi esclarcissant par façon que la lune n'avoit clarté qui peust plus le cler jour surmonter, et ja les oiselets s'appeloient l'un l'autre. D'autre part la mer estoit coye et sereine et ne bruyoit en façon nulle ne que fist un estang. Les mouettes aussi commencerent à voler par dessus la marine, et d'autres si trottoient sur le sablon menu que beau les faisoit veoir. Le jour tant s'efforça qu'il envoya coucher la lune et les estoiles, si que plus nulles ou ciel n'apparaissoit. (id.)

'Alors ils virent l'air net, pur, sans vent et sans nuage, et le jour l'emportait sur la nuit, s'éclaircissant de telle sorte que la lune n'avait plus de clarté qui puisse dépasser celle du jour, et déjà les oiselets s'appelaient. D'un autre côté, la mer était tranquille et sereine et ne faisait pas plus de bruit qu'un étang. Les mouettes aussi commencèrent à voler sur la plage, et d'autres aussi marchaient sur le sable fin; c'était beau à voir. Le jour fit tant d'efforts qu'il envoya la lune se coucher, ainsi que les étoiles, de sorte que nulle n'apparaissait dans le ciel.'

Ce type de progression, qui, dans cet exemple, s'accompagne encore de l'emploi de quelques formes de passé simple (commencèrent, s'efforça), comme des sortes de traces de la primauté du premier plan sur le second plan, apparait fréquemment dans les textes argumentatifs; il ne s'agit évidemment plus d'opposer un déroulement chronologique à un arrière-plan plus statique, mais de développer par exemple une affirmation générale en plusieurs énoncés plus "particuliers", ou d'illustrer une thèse par diverses propositions successives qui vont dans le même sens, etc. Dans l'extrait suivant, l'enchaînement des phrases se fait sur le schéma syntaxique SVO, l'hyperthème, sousentendu par la première phrase, renvoyant à la situation, pour le moins difficile, de la 
France au début du XVème siècle : chacune des phrases, dans sa globalité - et non seulement le groupe sujet - constitue ainsi une sous-partie du développement, de l'illustration de ce thème général :

Et toi, pour quoi veux tu vieillir en telle male méchéance, et vivre en souhaitant la mort tous les jours? La chevalerie de ton pays est périe et morte ; les études sont dissipées ; le clergé est dispersé et vague et opprimé ; les citoyens sont dépourvus d'espérance; l'ordre est tourné en confusion et loi en démesurée violence ; juste seignerie et honneur déchoit; obéissance ennuie ; pacience faut ; tout tombe et fond en l'abisme de ruine et désolation.

(Alain Chartier, Le Livre de l'Espérance, XV ${ }^{\text {ème }} \mathrm{s}$.)

13 La cohérence n'étant plus assurée par la présence d'indices formels, par l'utilisation d'un système de coréférence, on comprend que l'ordre direct, non marqué, soit le plus apte à traduire cette progression; on comprend aussi qu'apparaîtront de plus en plus fréquemment, dans une telle perspective, les cas de "rupture » thématique; du moment que la cohérence s'effectue sans éléments de liaison, sans rattachement à un hyperthème explicitement énoncé, l'ordre direct sera exploité pour introduire un nouvel épisode dans le texte, un référent ou un événement que rien n'annonce, d'une façon ou d'une autre, dans le contexte. C'est alors l'ordre du texte seul qui maintient la cohérence.

Par cet examen bien trop rapide de la notion textuelle de «plan » en ancien et en moyen français, nous avons voulu montrer que les catégories de la typologie syntaxique, en l'occurrence ici celles qui concernent l'ordre des constituants (schéma non marqué, rôle de la construction à verbe second, etc.) peuvent être corrélées à des phénomènes qui relèvent de la cohérence du texte, ce rapprochement s'avérant même indispensable si l'on désire dépasser le cadre des simples constatations. Il semble toutefois difficile de ne pas tenir compte de la structuration et de l'évolution des catégories textuelles ellesmêmes : parler de second plan comme s'il s'agissait d'une notion universelle, dont ne varierait, finalement, que les marques fournies par les systèmes linguistiques particuliers, masque le fait qu'il y a, au niveau textuel, plusieurs conceptions du second plan, conceptions qui se rattachent d'ailleurs à des «intuitions » différentes de la cohérence (cohérence "étroite» vs cohérence "large", par exemple). Essayer de montrer les spécificités du domaine textuel en s'appuyant, comme on le fait souvent, sur les différences dues à la situation de communication (oral vs écrit), ou sur le jeu des diverses fonctions du langage (fonction référentielle vs fonction «fictionnelle »), n'est sûrement pas suffisant. Il s'agit toujours, au bout du compte, d'une démarche qui rattache "directement » le type de texte, défini sans doute avec de plus en plus de précision, aux faits de langue ; cette simplification présente l'inconvénient de ne pas prendre en compte le niveau intermédiaire, celui du système des notions textuelles, qui possède sa structuration propre et dans lequel s'opère, avec ses contraintes particulières, le codage linguistique qui assure la cohérence du texte. 


\section{BIBLIOGRAPHIE}

DRESSLER, W. (1996) «Parallelisms between Natural Textlinguistics and other components of Natural Linguistics », Sprachtylopologie und Universalienforschung, 49, 295-311

DU BoIs, J.W. (1987) « The discourse basis of ergativity », Language, 63, 805-855

DURIE, M. (1994) « A case study of pragmatic linking », Text, 14, 495-529

GIVON, T. (1992) « The grammar of referential coherence as mental processing instructions », Linguistics, 30, 5-55

HopPeR, P. (1985) » Discourse Function and Word Order Shift : A Typological Study of the VS / SV alternation », in W.P. Lehman, éd., Language Typology 1985, J. Benjamins, 123-140

HOPPER, P. et THOMPSON, S.A. (1980) « Transitivity in grammar and discourse », Language, 56, 251-299

HOPPER, R. (1998) « Universals of narrative pragmatics : a Polynesian case study », Linguistics, 36, $119-160$

MYHILL, J. (1992) Typological Discourse Analysis : quantitative approaches to the study of linguistic function, Blackwell

O'DowD, E. (1990) « Discourse pressure, genre and grammatical alignment - after Dubois », Studies in Language, 14, 365-403

PAYNE, D. (1987) «Information structuring in Papago narrative discourse », Linguistics, 63, 782-804

PAYNE, D. (1994) « OVSu versus VSuO in Panare (Cariban) : Do syntax and discourse match?», Text, $14,581-610$

PRINCE, E.F. (1988) « Discourse analysis : a part of the study of linguistic competence », in Linguistics : The Cambridge Survey, vol.II, 164-182

REINHART, T. (1984) «Principles of gestalt perception in the temporal organization of narrative texts ", Linguistics, 22, 779-809

\section{AUTEUR}

\section{BERNARD COMBETTES}

Université Nancy II 